\title{
Liposomal Eye Spray Is as Effective as Antihistamine Eye Drops in Patients with Allergic Rhinoconjunctivitis Induced by Conjunctival Provocation Testing
}

\author{
Anne-Nele Grzella ${ }^{a}$ b Sabine Schleicher ${ }^{a}$ Kija Shah-Hosseini ${ }^{a}$ \\ Anatoli Astvatsatourov $^{c}$ Esther Raskopf ${ }^{b}$ Silke Allekotte ${ }^{b}$ Ralph Mösges ${ }^{a, b}$

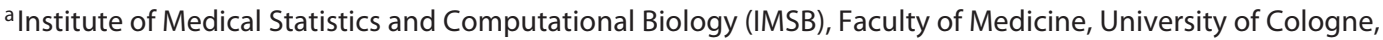 \\ Cologne, Germany; ${ }^{b} \mathrm{CRI}$ - Clinical Research International Ltd., Cologne, Germany; ${ }^{\mathrm{c} C l i n i c a l}$ Trials Center Cologne, \\ Faculty of Medicine, University of Cologne, Cologne, Germany
}

\section{Keywords}

Allergic rhinoconjunctivitis · Conjunctival provocation test · Digital analysis · Liposomal eye spray · Nonpharmacological treatment

\footnotetext{
Abstract

Background: Liposomal eye spray (LS) has been introduced for the treatment of dry eye. Whether LS can also relieve symptoms due to allergic rhinoconjunctivitis (ARC) has not yet been sufficiently investigated. Objectives: The aim of this study was to assess the onset of action, the reduction of allergy symptoms, and the safety of LS - a nonpharmacological treatment option - compared with those of antihistamine eye drops (AD). Methods: In this open, prospective, controlled, monocenter noninterventional study, adults with $A R C$ received either $L S$ or $A D$ for the relief of eye irritation after a positive conjunctival provocation test (CPT). All patients completed a questionnaire before and after the CPT. Eye irritation was rated on a visual analogue scale (VAS) at 6
}

\begin{tabular}{ll}
\hline KARGER & ( 2019 S. Karger AG, Basel \\
E-Mail karger@karger.com & $\begin{array}{l}\text { This article is licensed under the Creative Commons Attribution- } \\
\text { NonCommercial-NoDerivatives 4.0 International License (CC BY- } \\
\text { www.karger.com/iaa }\end{array}$ \\
$\begin{array}{l}\text { NC-ND) (http://www.karger.com/Services/OpenAccessLicense). } \\
\text { Usage and distribution for commercial purposes as well as any dis- } \\
\text { tribution of modified material requires written permission. }\end{array}$
\end{tabular}

time points. Conjunctival redness was analyzed using objective digital analysis based on images taken during the study visit. Results: Data were collected from 40 patients (20 per group). In both groups, $80 \%$ of patients perceived an onset of action within 0-2 min after application of LS or AD following the CPT. Relief of eye irritation (as determined by VAS) increased throughout the visit for both groups. In the digital analysis, the mean proportion of redness of the eye decreased from 10.3 to $7.0 \%$ for LS and from 10.4 to $6.5 \%$ for AD, with the largest difference observed 10 min after application (LS: 8.9\%; AD: 6.0\%; $p=0.094$ ). Conclusions: LS is a nonpharmacological treatment option for $A R C$, showing no significant difference or relevant numerical inferiority to AD in any parameter studied. It was generally safe and well tolerated.

(c) 2019 S. Karger AG, Basel
Prof. Dr. med. Dipl--Ing. Ralph Mösges

Institute of Medical Statistics and Computational Biology (IMSB) Faculty of Medicine, University of Cologne

Kerpener Strasse 62, DE-50937 Cologne (Germany)

E-Mail ralph@moesges.de 


\section{Introduction}

With a prevalence of $10-20 \%$, allergic rhinoconjunctivitis (ARC) is one of the most common disorders of the upper respiratory tract, causing not only nasal and ocular symptoms, but also a decline in quality of life $[1,2]$. Seasonal ARC is caused by different pollens or molds, whereas perennial allergic rhinitis is associated with allergens from house dust mites, molds, or animals [2].

Guidelines recommend the application of local or oral antihistamines or nasal glucocorticoids for the symptomatic treatment of ARC [3]. Topical antihistamines such as levocabastine, which is a component of LIVOCAB direkt ${ }^{\circledR}$ antihistamine eye drops (AD), are preferably applied in ARC $[4,5]$. Levocabastine is a second-generation H1 receptor antagonist.

As an alternative treatment option to local antihistamines, the liposomal eye spray (LS) Tears Again ${ }^{\circledR}$ (Optima Pharmazeutische $\mathrm{GmbH}$, Hallbergmoos, Germany) has been introduced [6]. The liposomes contained in this spray are applied to the closed eyelid and blend with the lipids secreted by the meibomian glands at the rim of the eyelids. Upon opening the eye, the lipids spread into the tear film. The lipids supplement the destabilized tear film, thus protecting the conjunctival mucous membrane.

The stabilizing effect of LS for the treatment of dry eye has been confirmed in several studies [7-12]: In a prospective randomized double-masked investigation, Craig et al. [7] reported significant improvements in lipid characteristics, such as tear film stability and lipid layer thickness as well as improved comfort, in patients after the application of LS. Furthermore, results of prospective controlled open studies show that liposomes can be applied as a nasal spray and thus can be successfully used to treat rhinitis symptoms [13-15]: Similar to the effect on the conjunctiva, the liposomes integrate into the destabilized "nasal surfactant" and stabilize the natural moisture film [16]. According to Weston and Mösges [15], the liposomal nasal spray reduced symptoms and significantly improved quality of life in patients with ARC, showing no difference to standard treatment with an antihistamine and/or glucocorticoid spray, as was shown in a prospective, controlled, open, observational study. To assess the efficacy and tolerability in patients with seasonal ARC, Böhm et al. [6] compared in a prospective, controlled, open, observational study the use of a liposomal nasal spray in combination with an LS to that of a standard cromoglycate-based combination therapy. The use of the liposomal products led to significant symptom relief, underlining the potential of liposome-based therapy and warranting further research. Especially patients who have reservations about pharmacological products or who have had unsatisfactory results with conventional therapy might prefer nonpharmacological treatment options [17].

Overall, previous results from prospective, controlled studies (some of which were randomized and blinded or open-label) suggest that liposomes, in particular in the form of an eye spray, are a promising alternative to standard drug therapy for treating allergic symptoms and should be investigated further [6-12]. Consequently, it was the objective of the present study to gain information on how stabilization of the tear film can influence eye symptoms/irritation during natural allergen exposure in the allergy season. Therefore, we analyzed liposomes as a potential nonpharmacological treatment option to topical antihistamines by assessing their respective onsets of action as well as their capability of reducing allergy symptoms after a conjunctival provocation test (CPT). Through artificial allergen exposure, the CPT serves as a model for the allergy season by triggering typical symptoms of ARC $[18,19]$. Current guidelines recommend carrying out the $\mathrm{CPT}$ either before initiating allergen immunotherapy (AIT) to determine the allergens which induce the symptoms or conducting the CPT throughout the course of AIT to monitor its therapeutic effect [20].

\section{Methods}

Study Design

The study was planned as an open, prospective, controlled, monocenter, noninterventional study and was conducted in an otorhinolaryngology practice specialized in allergy in Aachen, Germany, from January to February 2017. It consisted of one visit at which a CPT was performed and either AD or LS was administered. Patients completed a questionnaire before and after the $\mathrm{CPT}$, and they also rated the relief of eye symptoms on a visual analogue scale (VAS). Digital images were taken to track the changes of the eye mucosa after administration of $\mathrm{AD}$ or LS. The digital images where subsequently analyzed by objective digital analysis and external observers. A timeline of the study visit is shown in Figure 1. A follow-up telephone call to the patients was carried out $6-12 \mathrm{~h}$ after the study visit to document the occurrence of any adverse events or serious adverse events.

\section{Study Population}

Forty patients were planned to be enrolled in this study. All patients had to meet the following inclusion criteria: a history of ARC triggered by birch pollen, grass pollen, or house dust mites, and the necessity to perform a CPT for either diagnostic confirmation prior to the start of an AIT or to monitor the progress throughout the course of an AIT. All patients had to have a positive CPT (stage II or higher, according to Table 1) in order to be enrolled in the study. Exclusion criteria related to the CPT were allergy symp- 


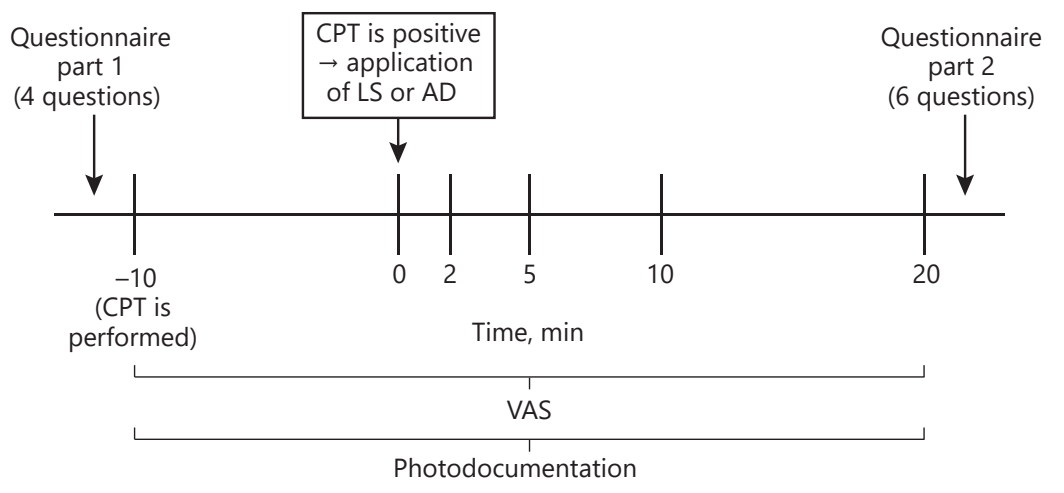

Fig. 1. Timeline of the study visit: Part 1 of the questionnaire was completed before the CPT was performed. Immediately after the CPT had been assessed as positive, either LS or AD were administered. The VAS was scored at 6 time points (after the positive CPT as well as $0,2,5,10$, and $20 \mathrm{~min}$ after application of LS or AD).
Images were taken at these 6 time points as well as before the CPT (control). Part 2 of the questionnaire was completed after VAS and photodocumentation. AD, antihistamine eye drops; CPT, conjunctival provocation test; LS, liposomal eye spray; VAS, visual analogue scale. toms already present at the time of the CPT, other ocular disorders except for refraction anomalies, wearing of contact lenses for the $72 \mathrm{~h}$ prior to the $\mathrm{CPT}$, and ongoing antiallergic therapy that might interfere with the CPT [19].

\section{Questionnaire}

The questionnaire was divided into two parts (Fig. 1). Part 1 consisted of four questions and was to be completed by the patients prior to undergoing the CPT. The questions aimed at capturing the current state of the eyes: pain or discomfort, urge to rub the eyes, vision, and reading difficulties. All questions provided four possible response options, ranging from good to poor. Twenty minutes after the CPT, patients had to complete part 2 of the questionnaire. Besides answering the same four questions from part 1, patients were also asked to assess the onset of action and the relief of eye irritation after using the respective product. The possible response options for the onset of action were $0-2,3-5,6-10$, and 11-20 min. The relief of eye irritation was to be rated as "very good," "good," "moderate," or "poor."

\section{Conjunctival Provocation Test}

After ensuring that the patients met all inclusion criteria and did not fulfill any of the exclusion criteria, the CPT was performed according to Riechelmann et al. [21] using the standardized, high-quality ALK-lyophilized allergen extracts of birch, grass, or house dust mite (ALK-Abelló A/S, Hørsholm, Denmark). Dilutions were prepared as described in the manufacturer's instructions. The CPT was considered positive if the allergic reaction was assessed as being stage II or higher (Table 1). The patients chose their favorite treatment option after having received a detailed explanation of the respective products from their physician. Then, AD or LS were administered (in accordance with the patient's decision) immediately after a positive result to reduce eye symptoms.

Liposomal Eye Spray for Symptomatic

Treatment of Rhinoconjunctivitis
Table 1. Categorization of the response to the allergen in the CPT according to Riechelmann et al. [21] (stage II or higher is considered positive)

\begin{tabular}{ll}
\hline Stage & Criteria \\
\hline 0 & $\begin{array}{l}\text { no subjective or visible reaction } \\
\text { itching, reddening, foreign body sensation } \\
\text { I }\end{array}$ \\
II & $\begin{array}{l}\text { cone I and in addition tearing, vasodilation of the } \\
\text { stage II and in addition vasodilation and erythema of } \\
\text { the conjunctiva tarsi, blepharospasm } \\
\text { stage III and in addition chemosis, lid swelling }\end{array}$ \\
III & IV
\end{tabular}

CPT, conjunctival provocation test.

\section{VAS Score}

Patients rated the relief of eye irritation on a VAS at 6 time points: immediately after the CPT as well as directly (0 min), 2, 5, 10 , and $20 \mathrm{~min}$ after the application of $\mathrm{AD}$ or LS. The VAS ranged from "not at all comfortable" (0\%) to "very comfortable" (100\%).

\section{Photodocumentation and Digital Analysis}

To objectively assess the redness of the eye, digital images of the provoked eye were taken at 7 time points throughout the study visit: before the CPT (control), immediately after a positive CPT, and also each time the VAS was rated. The photodocumentation set consisted of a foldable stand and a digital camera with specific preset configuration parameters as described in Astvatsatourov et al. [22]. The technical settings were consistent throughout the 
study. The digital images were analyzed by means of objective digital analysis according to Astvatsatourov and Mösges [23, 24]. Using image segmentation and pattern recognition, the redness of the conjunctiva was determined, which was defined as the percentage of red pixels in the region of interest.

\section{External Observer}

Besides undergoing digital analysis, the images were also assessed by two blinded independent external observers. Redness was classified according to its severity: $0=$ no redness, $1=$ mild redness, $2=$ moderate redness, $3=$ severe redness. The assessments of both external observers were compared, and any discrepancies were again rated jointly by both observers.

\section{Statistical Analysis}

The sample size was calculated under the assumption that $50 \%$ of patients in one group and $90 \%$ of patients in the second group would perceive relief of eye irritation for the first time $5 \mathrm{~min}$ after application. Given a $5 \%$ error and a power of $80 \%$, a group size of 20 patients was estimated. Statistical analyses were performed with IBM SPSS Statistics for Windows Version 25.0 (IBM Corp., Armonk, NY, USA). Double data entry was carried out to reduce data entry errors and the data were checked for plausibility. Missing entries for the VAS score were substituted with the "last value carried forward" method. All other missing entries were treated as "missing values." Continuous data were described as means, standard deviations, minimums, maximums, and number of valid cases. Categorical data were described as absolute and relative frequencies. Any differences between the two treatment groups in the VAS score and the digital analysis were analyzed using the Wilcoxon-Mann-Whitney test. A $p$ value $<0.05$ was considered statistically significant.

\section{Results}

\section{Demographic Data and CPT Results}

Forty patients aged 20-62 years (mean $35 \pm 13.9$ years) were included in the study; 26 patients were female and 14 were male. Each study group consisted of 20 patients. Twenty-five patients wore glasses, and none were contact lens wearers. Comorbidities or concomitant medication were not documented in this study. All patients had a history of ARC (triggered by birch pollen, grass pollen, or house dust mites), but did not show any symptoms upon inclusion in the study. All 40 patients had a positive CPT (stage II) as rated by the physician.

\section{Safety Aspects}

No treatment-related adverse events or serious adverse events occurred during the study. One patient treated with $\mathrm{AD}$ experienced an adverse event (self-limited mild epistaxis) that had no correlation with the treatment.

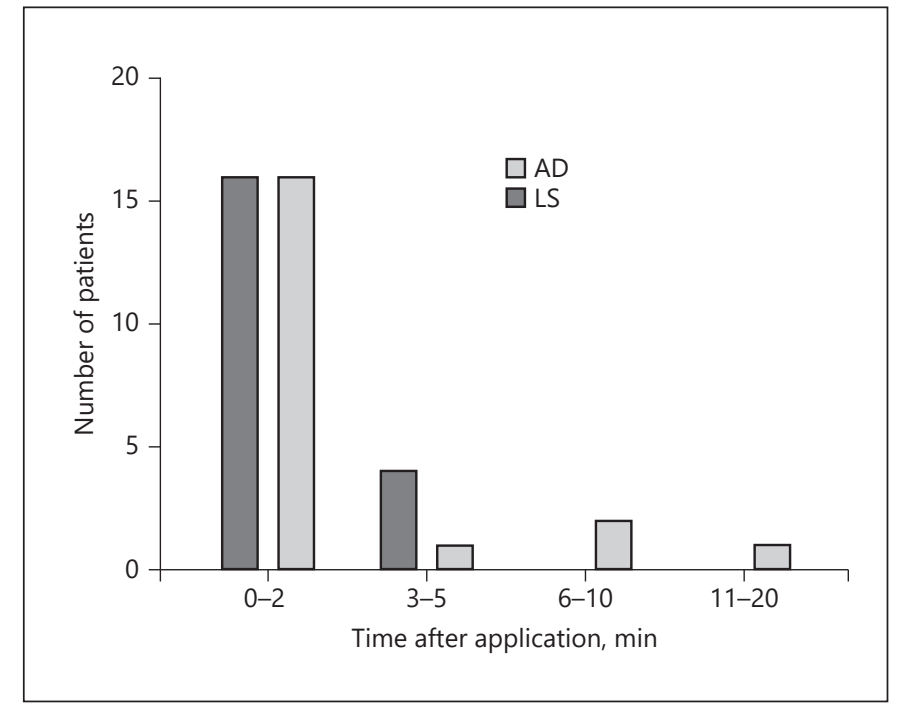

Fig. 2. Onset of action after administration of $\mathrm{AD}(n=20)$ or LS $(n=20)$, defined as the first perception of relief after eye irritation. $\mathrm{AD}$, antihistamine eye drops; LS, liposomal eye spray.

\section{Onset of Action}

In each study group, $80 \%$ of the patients ( 16 per group) perceived an onset of action, being defined as the first perception of symptom relief, within $0-2$ min after receiving their respective treatment (Fig. 2). In the LS group, 4 patients felt an onset of action within 3-5 min. In contrast, 1 patient in the AD group felt an onset of action within 3-5 min, 2 patients within 6-10 min, and 1 patient within 11-20 min. No significant differences were found between the two study groups.

\section{Questionnaire}

The questions regarding pain or discomfort, the urge to rub the eye, vision, and reading difficulties asked prior to and after the CPT showed no significant differences between the two study groups. The relief of eye irritation was rated as "very good" by 6 patients in the LS group and 7 patients in the AD group. Nine patients in the LS group and 10 patients in the AD group gave the rating "good." Relief was rated as "moderate" by 5 patients in the LS group and by 3 patients in the $\mathrm{AD}$ group. None of the patients in either group gave the rating "poor."

\section{VAS Score after Application of LS and AD}

The mean VAS score for eye irritation relief showed an overall similar increase in both groups. The score increased from $27.4 \%$, rated immediately after a positive reaction in the CPT, to $71.9 \% 20 \mathrm{~min}$ after application of LS, 


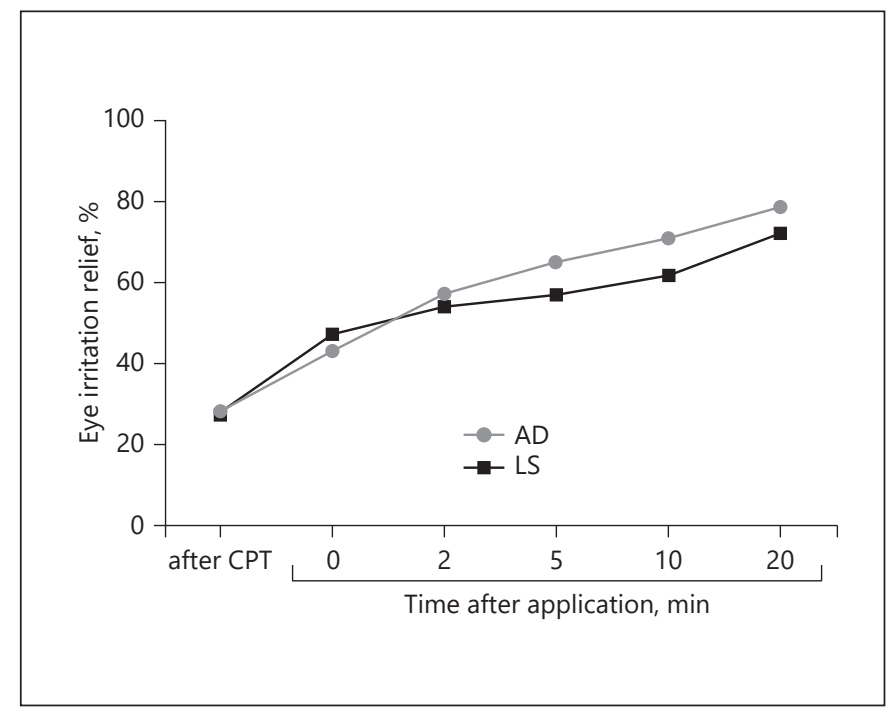

Fig. 3. Patients rated the relief of eye irritation at 6 time points (after positive CPT as well as $0,2,5,10$, and 20 min after application) throughout the study using the VAS, which ranged from "not at all comfortable" (0\%) to "very comfortable" (100\%). Data are presented as mean percentages of the VAS. AD, antihistamine eye drops; CPT, conjunctival provocation test; LS, liposomal eye spray; VAS, visual analogue scale.

and from 28.3 to $78.9 \%$ for the $\mathrm{AD}$ group, respectively (Fig. 3). Even though the mean score for AD was slightly higher than that for LS, no significant difference was found between the treatment groups at any time point.

\section{Digital Analysis of Conjunctival Redness}

The objective digital analysis of the images taken during the study visit showed a reduction in the percentage of red pixels in the region of interest and therefore a decrease in redness of the eye for both treatment groups (Fig. 4). Immediately after the CPT, redness was $9.4 \%$ for LS and $7.9 \%$ for AD. The highest mean percentage of eye redness was observed directly after treatment application, with $10.3 \%$ being noted for $\mathrm{LS}$ and $10.4 \%$ for $\mathrm{AD}$. At the end of the observation period (20 min after treatment), the mean percentage had decreased to $7.0 \%$ for $\mathrm{LS}$ and to $6.5 \%$ for $\mathrm{AD}$. The largest difference between the two treatment groups was observed $10 \mathrm{~min}$ after application (LS: $8.9 \%$; AD: $6.0 \%)$. There was a trend $(p=0.094)$ towards a more pronounced action of $\mathrm{AD}$ at this time point. Overall, no significant differences were observed at any time point.

\section{Assessment by External Observers}

The assessments made by the external observers $(0=$ no redness, $1=$ mild redness, $2=$ moderate redness, $3=$

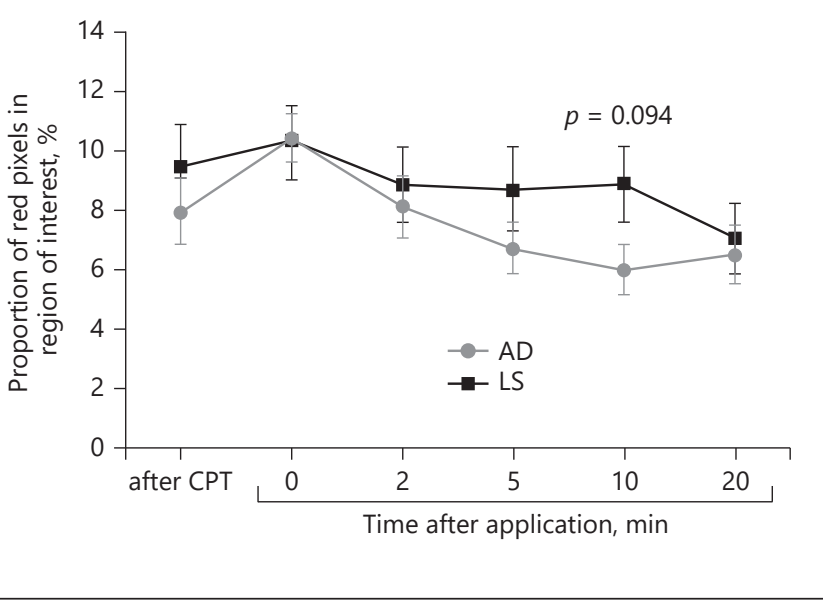

Fig. 4. Objective digital analysis of the redness of the eye was performed for digital images taken at 7 time points (before CPT [control], after CPT, as well as 0,2, 5, 10, and $20 \mathrm{~min}$ after application) during the study visit. Data are presented as mean \pm SEM. AD, antihistamine eye drops; CPT, conjunctival provocation test; LS, liposomal eye spray.

severe redness) showed similar results for both treatment groups, with only a slight change in the mean value being noted between baseline (LS: 1.3; AD: 1.15) and $20 \mathrm{~min}$ after application (LS: 1.15; AD: 1.15). For both groups, the most frequent assessment at any time point was mild redness, followed by moderate redness, no redness, and severe redness (Fig. 5). No significant difference was observed between the study groups.

When comparing the results from the digital analysis with those from the assessments by the external observers, a linear correlation was observed for AD with a Pearson correlation coefficient of 0.42 .

\section{Discussion and Conclusion}

This study is the first to compare LS with $\mathrm{AD}$ in patients with ARC. The aim of this study was to determine the onset of action and the reduction of allergy symptoms for the tested nonpharmacological LS following allergen exposure as compared to those for guideline-recommended treatment with $\mathrm{AD}$. The allergic reaction was triggered by conjunctival provocation, which has been shown to serve as a predictive model for ARC symptoms during the allergy season [18]. 
Fig. 5. Assessment of eye redness by the external observer using the images from photodocumentation $(0=$ no redness, $1=$ mild redness, $2=$ moderate redness, $3=$ severe redness). $\mathrm{AD}$, antihistamine eye drops; CPT, conjunctival provocation test; LS, liposomal eye spray.

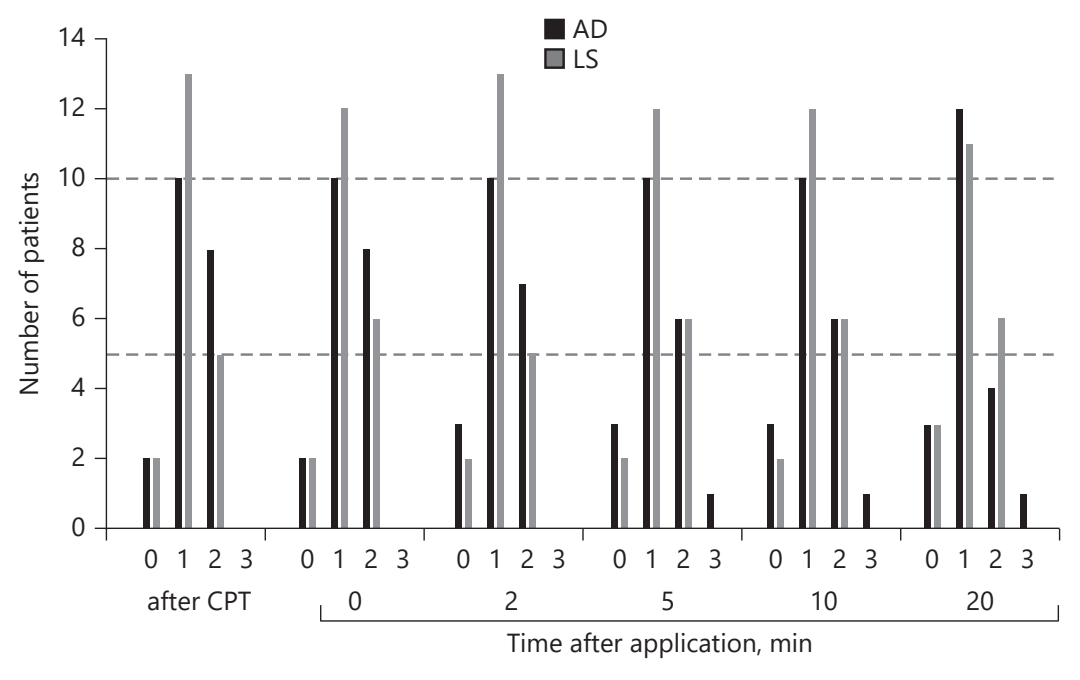

Since ARC is a common condition all over the world, treatment options have been thoroughly investigated in multiple studies, and guidelines for an optimal approach have been established $[1,2]$. These guidelines recommend the administration of oral antihistamines or leukotriene antagonists, or the application of topical antihistamines or nasal glucocorticoids for the symptomatic treatment of allergy symptoms. While many patients use these conventional therapies, some patients have reservations about pharmacological products and tend to try alternative options, including homeopathy or acupuncture. According to a survey conducted by Schäfer et al. [17], 26.5\% of patients used alternative medicine for their allergies. Their reasons for doing so were unsatisfactory results from conventional therapy, the belief that alternative medicine has fewer side effects, and the desire to "try everything" to reduce allergy symptoms. Given these findings, medical products with liposomes - such as LS - might be especially interesting for patients who do not want to apply pharmaceutical medication for fear of side effects or interactions, but who desire similar symptom relief. Regarding LS, its mode of action is not based on pharmacological principles. The liposomes contained in it consist of phospholipids that also make up parts of the lipid layer of the tear film and thus play an important role in the proper functioning of the tear film. Consequently, liposomes can be used to stabilize the tear film by complementing the lipid layer. Furthermore, liposomes have been found to reduce in- flammation of the rim of the eyelid in patients suffering from dry eye [11].

An important aspect for patients suffering from allergic symptoms is not only the overall efficacy of a product, but also its onset of action. This noninterventional study showed that $\mathrm{LS}$ was as effective as $\mathrm{AD}$ regarding the onset of action. In both treatment groups, $80 \%$ of patients perceived the onset of action within 0-2 min after application, resulting in the first perception of relief of allergy symptoms. This finding goes in line with that of an earlier prospective, controlled, open, observational study conducted by Böhm et al. [6], which compared a cromoglycate combination therapy to a liposomal nasal spray, either alone or in combination with an LS. They found that $25 \%$ of LS users felt an onset of action within $5 \mathrm{~min}$, whereas only $12.5 \%$ of cromoglycate eye drop users perceived an onset of action within the same time frame. Furthermore, the liposomal nasal spray in combination with the LS and the cromoglycate combination therapy were safe and well tolerated. Similar to the findings by Böhm et al. [6], the safety and tolerability of the treatment investigated here can be assessed as good, since no treatment-related adverse events or serious adverse events occurred. These results are consistent with those from previous studies evaluating the effect of LS for dry eye $[8,10]$ and with findings from studies assessing liposomal nasal spray $[13,15]$.

In addition to objective assessments, the tolerability and efficacy of study treatments can also be evaluated using information regarding overall patient satisfaction. Patient 
satisfaction was assessed on the basis of a VAS score, ranging from "not at all comfortable" $(0 \%)$ to "very comfortable" (100\%). The score for AD increased from $28.2 \%$ (immediately after the CPT) to $78.9 \%$ (20 min after application), suggesting good efficacy. For LS, a comparable increase from 27.4 to $71.9 \%$ was observed, indicating considerable relief of eye irritation and further demonstrating the noninferiority of LS. The results of the patients' perception of the treatment are comparable to those reported in studies exploring the application of liposomes for dry eye and in studies assessing liposomal nasal spray for treating seasonal allergic rhinitis. Subjective improvement of symptoms was observed in these studies $[6-8,11,14]$. For example, according to Lee et al. [11], 72\% of patients indicated noticeable relief of dry eye symptoms after the application of LS. Also, the application in form of a spray was considered by all patients to be more pleasant than the use of eye drops. Some patients might need to apply a product for relief of eye irritation every day. Therefore, the use of a spray instead of eye drops might be especially interesting for patients who need treatment regularly and who are uncomfortable with applying eye drops.

Not many studies exist that have evaluated the ocular application of liposomes for the treatment of ARC. A previous study assessing a liposomal nasal spray also showed a significant reduction in conjunctival symptoms after its application [15]. This finding shows that a nasal spray is capable of reducing eye symptoms, demonstrating the high efficacy of liposomal products.

The observation that the efficacy of LS is similar to that of standard therapy with $\mathrm{AD}$ confirms that LS is a suitable alternative for treating allergic eye symptoms. The results of the VAS score, the onset of action, and the questionnaires support that statement. Despite its pharmacological mode of action, no superiority was observed for AD as shown by the subjective patient evaluation.

Apart from $\mathrm{AD}$, there are other frequently used and well-established pharmaceutical products for the treatment of allergic conjunctivitis, such as nedocromil, emedastine, azelastine, and olopatadine, that have a similar onset of action. Overall study results suggest good clinical efficacy and a fast onset of action for these products [25-28]. In an open observational study of 102 patients with ARC, the mast cell stabilizer nedocromil was found to be well tolerated and showed no significant difference in symptom reduction when compared with levocabastine. Onset of action was perceived within the first hour by $82.9 \%$ of patients treated with nedocromil [26]. Emedastine is a second-generation H1 receptor antagonist. Netland et al. [27] described a rapid onset of action when this product was instilled 10 min or

Liposomal Eye Spray for Symptomatic

Treatment of Rhinoconjunctivitis
$2 \mathrm{~h}$ before conjunctival allergen challenge. Emedastine was more effective than levocabastine in inhibiting ocular itching and equivalent in inhibiting redness. Besides being an $\mathrm{H} 1$ antagonist, azelastine also has a mast cell stabilizing effect. In a randomized, active- and placebo-controlled study conducted by Bousquet et al. [25], patients received azelastine in combination with fluticasone while in an environmental exposure chamber. Onset of action of the active treatments tested (azelastine in combination with fluticasone, loratadine, and fluticasone alone) was defined as the first time point demonstrating a change from baseline which was greater than that for placebo and which continued until the last assessed time point. The total ocular symptom score for azelastine showed an onset of action $10 \mathrm{~min}$ after application, and the total nasal symptom score showed an onset of action after $5 \mathrm{~min}$. In another study, an $\mathrm{H} 1$ antagonist olopatadine formulation containing a vehicle was shown to stabilize mast cells and was found to be significantly better than its vehicle in reducing ocular itching at onset of action and 16 and $24 \mathrm{~h}$ after onset of action [28]. Similar to AD and LS, these pharmaceutical products showed a fast onset of action.

To objectively determine changes in eye irritation, redness was analyzed using digital imaging and analysis. Two external observers also rated the images. The digital images were taken at baseline before the CPT, immediately after a positive CPT, and at 5 time points after application of the respective product. The evaluation of redness by means of digital analysis showed a similar reduction in redness after application of $\mathrm{LS}$ or $\mathrm{AD}$. As expected, $\mathrm{AD}$ reduced the redness of the eye more strongly than did LS. This finding could be due to the pharmacological mechanism of action of AD. However, the difference was not significant. The digital analysis underlines the marginal differences between the products that were already observed in the VAS score. Thus, digital analysis presents itself as a very sensitive method for evaluating the redness of the eye. The benefit and purpose of digital analysis have been demonstrated in multiple studies $[29,30]$. This objective method for evaluating the relief of eye irritation further confirms the subjective patient evaluation and thus strengthens the hypothesis that $\mathrm{LS}$ is as effective as $\mathrm{AD}$ in reducing allergy symptoms. As assessed by the external observers, the redness of the eye decreased only slightly for both groups throughout the study visit, which might be due to the sometimes unsatisfactory quality of the digital images. Still, the noninferiority of LS was confirmed since there was no significant difference between the study groups.

A limitation of this study is the lack of blinding. Some patients might look more favorably upon the use of a rel- 
atively new alternative treatment option, whereas others might prefer the familiar standard drug therapy. Generally, blinding would make individual preferences irrelevant because the patients would not know which product they are using. However, blinding was not possible in this study since two different forms of application were used, eye spray and eye drops. To overcome this, a blinded double-dummy approach could have been applied, but this was not feasible here because of two reasons: Firstly, the placebo eye drops might have some inherent effects like rinsing of the eye. Regarding the double-dummy approach, the rinsing of the eye with the placebo eye drops as the first of the two interventions could lead to a washout of the allergens. This can result in false-positive effects of the investigational product, whereas a placebo eye spray would not have this rinsing effect when applied on the closed eye like LS. Secondly, in Germany, placebo, such as saline eye drops, is not allowed as a control in a study if a standard therapy is available (as defined by the German Medical Association [Bundesärztekammer] [1]).

Another apparent limitation was the quality of the digital images taken of the eye during the study visit. Although it was difficult for the external observers to distinguish differences between the images, digital analysis was able to produce satisfactory results. However, we recommend further improvement of digital imaging to ensure that highquality images are available for use in future studies. Lastly, the short duration of the study only allowed conclusions to be drawn for a single application of the products. Nevertheless, this one visit fulfilled the purpose of this study by producing results regarding the onset of action and symptom reduction. It might also be interesting to extend this study over more than one study visit and explore the efficacy of the tested treatments in a larger study population.

The onset of action and relief of eye irritation was perceived for LS within only a few minutes of its application following the CPT. The study results confirm the noninferiority of LS versus standard therapy with $\mathrm{AD}$ in patients suffering from ARC. Thus LS, with its good tolerability and safety, represents an equal nonpharmacological treatment option to guideline-recommended treatment with antihistamines. Since this study shows promising results, the next step is to investigate the tolerability and efficacy of LS during the allergy season.

\section{Acknowledgment}

We are grateful to Gena Kittel for her valuable editing and language editing assistance.

\section{Statement of Ethics}

The study was approved by the Ethics Committee of the Faculty of Medicine, University of Cologne, Cologne, Germany (reference number: 16-394). Written informed consent was given by all patients prior to their inclusion in the study.

\section{Disclosure Statement}

A.-N. Grzella, S. Schleicher, K. Shah-Hosseini, A. Astvatsatourov, and E. Raskopf have nothing to disclose. R. Mösges reports personal fees from ALK, grants from ASIT biotech, personal fees from allergopharma, personal fees from Allergy Therapeutics, grants and personal fees from Bencard, grants from Leti, grants, personal fees, and nonfinancial support from Lofarma, nonfinancial support from Roxall, grants and personal fees from Stallergenes, grants from Optima, personal fees from Friulchem, personal fees from Hexal, personal fees from Servier, personal fees from Klosterfrau, nonfinancial support from Atmos, personal fees from Bayer, nonfinancial support from Bionorica, personal fees from FAES, personal fees from GSK, personal fees from MSD, personal fees from Johnson \& Johnson, personal fees from Meda, personal fees and nonfinancial support from Novartis, nonfinancial support from Otonomy, personal fees from Stada, personal fees from UCB, nonfinancial support from Ferrero, grants from Bitop AG, grants from Hulka, personal fees from Nuvo, and grants from Ursapharm, outside the submitted work. S. Allekotte reports grants and personal fees from Lofarma, personal fees from Servier, personal fees from Hexal, and personal fees from Friulchem, outside the submitted work.

\section{Funding Sources}

The study was funded by Optima Pharmazeutische GmbH, Hallbergmoos, Germany.

\section{Author Contributions}

A.-N. Grzella, S. Allekotte, and R. Mösges contributed to the study conduct and writing of the manuscript. S. Schleicher contributed to the study conduct. K. Shah-Hosseini and A.-N. Grzella did the statistical analysis. A. Astvatsatourov and A.-N. Grzella performed and evaluated the digital analysis. E. Raskopf contributed to the writing of the manuscript.

\section{References}

1 Bauchau V, Durham SR. Prevalence and rate of diagnosis of allergic rhinitis in Europe. Eur Respir J. 2004 Nov;24(5):758-64.

2 Brozek JL, Bousquet J, Baena-Cagnani CE, Bonini S, Canonica GW, Casale TB, et al.; Global Allergy and Asthma European Network; Grading of Recommendations Assessment, Development and Evaluation Working Group. Allergic Rhinitis and its Impact on Asthma (ARIA) guidelines: 2010 revision. J Allergy Clin Immunol. 2010 Sep;126(3):466-76. 
3 Bachert C, Borchard U, Wedi B, Klimek L, Rasp G, Riechelmann H, et al. [Allergic rhinoconjunctivitis. Guidelines of the DGAI in association with the DDG]. J Dtsch Dermatol Ges. 2006 Mar;4(3):264-75.

4 Rimås M, Kjellman NI, Blychert LO, Björkstén B. Topical levocabastine protects better than sodium cromoglycate and placebo in conjunctival provocation tests. Allergy. 1990 Jan;45(1):18-21.

5 Zuber P, Pécoud A. Effect of levocabastine, a new $\mathrm{H} 1$ antagonist, in a conjunctival provocation test with allergens. J Allergy Clin Immunol. 1988 Oct;82(4):590-4.

6 Böhm M, Avgitidou G, El Hassan E, Mösges R. Liposomes: a new non-pharmacological therapy concept for seasonal-allergic-rhinoconjunctivitis. Eur Arch Otorhinolaryngol. 2012 Feb;269(2):495-502.

7 Craig JP, Purslow C, Murphy PJ, Wolffsohn JS. Effect of a liposomal spray on the pre-ocular tear film. Cont Lens Anterior Eye. 2010 Apr;33(2):83-7.

8 Dausch D, Lee S, Dausch S, Kim JC, Schwert G, Michelson W. [Comparative study of treatment of the dry eye syndrome due to disturbances of the tear film lipid layer with lipidcontaining tear substitutes]. Klin Monatsbl Augenheilkd. 2006 Dec;223(12):974-83.

9 Khaireddin R. [Contact lens associated dry eye. Current study results and practical implementation]. Ophthalmologe. 2013 Jun; 110(6):511-4.

10 Khaireddin R, Schmidt KG. [Comparative investigation of treatments for evaporative dry eye]. Klin Monatsbl Augenheilkd. 2010 Feb; 227(2):128-34.

11 Lee S, Dausch S, Maierhofer G, Dausch D. [A new therapy concept for the treatment of dry eye - the usefulness of phospholipid liposomes]. Klin Monatsbl Augenheilkd. 2004 Oct;221(10):825-36.

12 Pult H, Gill F, Riede-Pult BH. Effect of three different liposomal eye sprays on ocular comfort and tear film. Cont Lens Anterior Eye. 2012 Oct;35(5):203-7.
13 Eitenmüller A, Piano L, Böhm M, Shah-Hosseini K, Glowania A, Klimek L, et al. Verträglichkeit und Auswirkungen auf die Lebensqualität durch die Behandlung mit einem liposomalen Nasenspray bei Patienten mit chronischer Rhinosinusitis. Allergologie. 2012;35(09):17-24.

14 Eitenmüller A, Piano L, Böhm M, Shah-Hosseini K, Glowania A, Pfaar O, et al. Liposomal nasal spray versus guideline-recommended steroid nasal spray in patients with chronic rhinosinusitis: A comparison of tolerability and quality of life. J Allergy (Cairo). 2014; 2014:146280.

15 Weston LA, Mösges R. Behandlung der saisonalen allergischen Rhinokonjunktivitis mit einem liposomalen Nasenspray. Allergologie. 2010;33(5):196-204.

16 Glowania A, Mösges R, Böhm M, Knopf A, Klimek L. Das Surfactant-System - ein neuer Therapieansatz für die Schleimhaut der oberen Atemwege. Atemw Lungenkrkh. 2011;37(10):1-5.

17 Schäfer T, Riehle A, Wichmann HE, Ring J. Alternative medicine in allergies - prevalence, patterns of use, and costs. Allergy. 2002 Aug;57(8):694-700.

18 Kruse K, Gerwin E, Eichel A, Shah-Hosseini K, Mösges R. Conjunctival provocation tests: a predictive factor for patients' seasonal allergic rhinoconjunctivitis symptoms. J Allergy Clin Immunol Pract. 2015 May-Jun;3(3):381-6.

19 Fauquert JL, Jedrzejczak-Czechowicz M, Rondon C, Calder V, Silva D, Kvenshagen BK, et al.; Interest Group on Ocular Allergy (IGOA) from the European Academy of Allergy and Clinical Immunology. Conjunctival allergen provocation test : guidelines for daily practice. Allergy. 2017 Jan;72(1):43-54.

20 Roberts G, Pfaar O, Akdis CA, Ansotegui IJ, Durham SR, Gerth van Wijk R, et al. EAACI guidelines on allergen immunotherapy: allergic rhinoconjunctivitis. Allergy. 2018 Apr; 73(4):765-98.

21 Riechelmann H, Epple B, Gropper G. Comparison of conjunctival and nasal provocation test in allergic rhinitis to house dust mite. Int Arch Allergy Immunol. 2003 Jan;130(1):51-9.
22 Astvatsatourov A, Reydelet Y, Mösges R. Photodocumentation of allergic severity under conjunctival provocation. Stud Health Technol Inform. 2015;213:11-4.

23 Astvatsatourov A, Mösges R. Image-based assessment of allergic inflammation under conjunctival provocation. Stud Health Technol Inform. 2015;213:15-8.

24 Astvatsatourov A, Mösges R. Specific characteristics in digital assessment of conjunctival redness. Stud Health Technol Inform. 2017; 238:181-4.

25 Bousquet J, Meltzer EO, Couroux P, Koltun A, Kopietz F, Munzel U, et al. Onset of action of the fixed combination intranasal azelastine-fluticasone propionate in an allergen exposure chamber. J Allergy Clin Immunol Pract. 2018 Sep-Oct;6(5):1726-1732.e6.

26 Kremer B, Tündermann A, Goldschmidt O. Onset of action, effectiveness and tolerance of levocabastine and nedocromil in topical therapy of seasonal allergic rhinoconjunctivitis. The Deutsche Rhinitis-Studiengruppe. Arzneimittelforschung. 1998 Sep;48(9):924-30.

27 Netland PA, Leahy C, Krenzer KL. Emedastine ophthalmic solution $0.05 \%$ versus levocabastine ophthalmic suspension $0.05 \%$ in the treatment of allergic conjunctivitis using the conjunctival allergen challenge model. Am J Ophthalmol. 2000 Dec;130(6):717-23.

28 Torkildsen G, Narvekar A, Bergmann M. Efficacy and safety of olopatadine hydrochloride $0.77 \%$ in patients with allergic conjunctivitis using a conjunctival allergen-challenge model. Clin Ophthalmol. 2015 Sep;9:1703-13.

29 Dogan S, Astvatsatourov A, Deserno TM, Bock F, Shah-Hosseini K, Michels A, et al. Objectifying the conjunctival provocation test: photography-based rating and digital analysis. Int Arch Allergy Immunol. 2014; 163(1):59-68.

30 Sárándi I, Claßen DP, Astvatsatourov A, Pfaar O, Klimek L, Mösges R, et al. Quantitative conjunctival provocation test for controlled clinical trials. Methods Inf Med. 2014;53(4): $238-44$.
Liposomal Eye Spray for Symptomatic

Treatment of Rhinoconjunctivitis
Int Arch Allergy Immunol 2019;179:123-131 DOI: $10.1159 / 000496938$ 